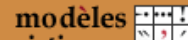

ling uistiques $\frac{1}{4}$ :

\section{Modèles linguistiques}

$59 \mid 2009$

Génétique de la production écrite et linguistique

\title{
L'écriture sur traitement de texte : quelques spécificités de son analyse en temps réel
}

\section{Claire Doquet-Lacoste}

\section{(2) OpenEdition \\ Journals}

Édition électronique

URL : https://journals.openedition.org/ml/341

DOI : $10.4000 / \mathrm{ml} .341$

ISSN : 2274-0511

Éditeur

Association Modèles linguistiques

Édition imprimée

Date de publication : 1 janvier 2009

Pagination : 133-151

Référence électronique

Claire Doquet-Lacoste, «L'écriture sur traitement de texte : quelques spécificités de son analyse en temps réel », Modèles linguistiques [En ligne], 59 | 2009, mis en ligne le 28 août 2013, consulté le 01 juillet 2021. URL : http://journals.openedition.org/ml/341 ; DOI : https://doi.org/10.4000/ml.341 


\section{L'écriture sur traitement de texte : quelques spécificités de son analyse en temps réel}

\section{Claire Doquet-Lacoste}

Activité absconse, généralement solitaire, émergeant dans l'intimité du scripteur et de sa plume, l'écriture se donne à voir aujourd'hui grâce à des outils informatiques qui permettent son enregistrement exhaustif et sa restitution en temps réel ${ }^{1}$. Ces données d'un nouveau type posent de multiples questions, à la fois à la linguistique et aux disciplines connexes, en particulier la génétique textuelle. Cette dernière, paradoxalement, demeure peu utilisée dans les analyses en temps qui ne s'appuient généralement qu'assez peu sur la nature linguistique des données (Favart \& Olive, 2005). Les outils de recueil conçus jusqu'ici l'ont été à des fins expérimentales et dans le cadre de recherches cognitives. Un instrument puissant de suivi du regard des scripteurs ${ }^{2}$, couplé à une tablette graphique, livre des informations inédites sur l'association entre le geste graphique et le regard, informations exploitées par les psychologues dans des descriptions fines des processus cognitifs. L'ensemble des études sur l'écriture en temps réel rend compte de données procédurales, à partir desquelles sont construits des modèles cognitifs faisant intervenir en particulier la mémoire de travail ${ }^{3}$; de ces études, les paradigmes linguistiques demeurent pratiquement absents.

Le présent article s'appuie sur des analyses linguistiques déjà réalisées à partir de corpus de reconstitutions d'écriture sur traitement de texte $^{4}$ ainsi que sur une recherche en cours à l'Item (pôle linguistique, équipe "Manuscrits, linguistique, cognition"). Il concerne la conservation et la lisibilité des traces de l'écriture sur traitement de texte, qui tend aujourd'hui à se généraliser et pour laquelle la linguistique n'a encore inventé que peu d'outils d'analyse.

1. Un des plus anciens est Genèse du texte, logiciel à finalité pédagogique développé en 1993 par l'Association Française pour la Lecture. Aujourd'hui, on peut citer Scriptlog (Université de Lünd, Suède) et Inputlog (Université de Anvers, Faculteit Toegepaste Economische Wetenschappen).

2. Dispositif Eye and Pen, développé à Poitiers (cf. Chesnet \& Alamargot 2005)

3. Cf. par exemple Alamargot \& Chanquoy, 2005 et Dedeyan \& al., 2006.

4. Doquet-Lacoste, 2004 \& 2006. 
A partir de corpus bruts, enregistrés informatiquement, nous poserons la question des données pertinentes pour la génétique textuelle et des modèles linguistiques permettant de les organiser pour les rendre lisibles.

\section{Des données nouvelles.}

Les données livrées par les outils de restitution de l'écriture en temps réel sont proprement fascinantes, dans tous les sens du terme. La restitution se fait d'abord sous forme de film, et face à l'écriture dans sa dynamique temporelle l'observateur est captivé par la succession des événements et l'empathie que suscite, comme au cinéma, la possibilité de suivre en temps réel le cheminement discursif. Il faut se garder de l'impression de penser avec le scripteur, sous peine de verser dans une appréhension subjective des faits linguistiques. Pour cette raison, ainsi bien sûr que pour la rigueur du traitement, il est fondamental de stabiliser les données mouvantes que constituent les « films de l'écriture »; la première question qui se pose au généticien souhaitant travailler sur le temps réel est donc celle de la transcription.

\subsection{Données accessibles sur des manuscrits / données accessibles avec les nouveaux outils.}

La génétique textuelle, forgée à l'occasion de l'étude de manuscrits, a développé deux types de transcription des données: la transcription linéaire et la transcription diplomatique. La transcription diplomatique tend à rendre l'image la plus fidèle possible du manuscrit en respectant sa mise en page: le transcripteur recopie le manuscrit en respectant la disposition des différents éléments, en notant les traits de biffure et leur étendue $^{5}$. La transcription chronologique, moins utilisée, est la reconstitution pas à pas des opérations d'écriture: le transcripteur interprète l'ordre chronologique des opérations selon leur place topographique, les ratures etc.; ce mode de transcription est rendu difficile à cause de l'hétérogénéité des traces et les données ne répondant pas à un ordre logiquement reconstituable, comme les listes par exemple ${ }^{6}$.

5. La transcription diplomatique est utilisée dans l'édition génétique, qu'elle soit scientifique ou de vulgarisation. Voir en particulier, comme un exemple très réussi d'édition génétique, le volume Récit d'Edmond Jabès. Les cinq états du mansucrit édité par M. Cohen, A. Crasson et I. Fenoglio (Cohen, Crasson \& Fenoglio, Paris, éd. Textuel, 2005).

6. Dans la transcription chronologique «on interprète la mise en place topographique comme trace d'un ordre chronologique affectant les opérations d'écriture et de traitement de l'écrit. Dans l'idéal, on reconstitue exhaustivement cet ordre. Dans la pratique, cette reconstitution rencontre des limites sérieuses :

- certaines données sont rebelles à la mise en ordre, soit qu'on ne dispose d'aucun indice pour la réaliser, soit qu'elle n'ait pas de sens. Je rappelle ici le cas des liste de mots utilisées par Joyce ou par V. Larbaud [...] 
Nous verrons par la suite quel type de transcription a été proposé pour les reconstitutions en temps réel, mais disons déjà que ces données comblent à l'avance certains «trous » des manuscrits :

Alors que l'établissement de la chronologie des opérations est un des objets de la recherche génétique à partir de manuscrits, cette chronologie est donnée exhaustivement et exactement dans les reconstitutions en temps réel. Par exemple, dans un manuscrit de ce type (écriture à l'école, extrait d'un texte d'élève de cours moyen 2 ) :

[...] Ensuite, toute la classe a commencés à marcher en dirrection de l'école où l'on nous attendaient pour passer la nuit et faire une boom méga géniale avee tous les copains copines. Nous avons même apris à Caroline à danser ; enfin, je pense.

Avant d'arriver nous nous sommes arrétés pour déjeuné.

Tout ce que montre le manuscrit de façon à peu près sûre, c'est que le deuxième paragraphe («avant d'arriver ») a été écrit après le premier. En revanche, aucun indice sur le moment où s'est faite la biffure. C'est ce qu'indique, en revanche, la reconstitution en temps réel, où l'on apprend qu'il s'est déroulé plus de 40 minutes entre l'inscription de ces énoncés et leur suppression, et que les deux propositions « Nous avons même appris à Carole à danser ; enfin, je pense » ont été supprimées avant le $\mathrm{SN}$ « et faire une boom méga géniale avec tous les copains et les copines ».

Alors que sur un manuscrit, les données concernant le rythme et la fluidité de l'écriture sont souvent des hypothèses, tirées de la nervosité d'un trait de plume ou de dessins en marge, les reconstitutions en temps réel indiquent l'ensemble des pauses effectuées par le scripteur. Ce faisant, elles permettent une description fine de la temporalité de l'écriture.

\subsection{Quelques problèmes posés par les données nouvelles.}

Ces données chronologiques et pausales sont évidemment extrêmement précieuses et permettent des investigations sur le processus d'écriture auxquelles les manuscrits ne donnent qu'imparfaitement accès. Toutefois, le risque existe que ces nouvelles données, par leur profusion et leur précision mêmes, noient l'essentiel de l'observable et empêchent d'y accéder. Un travail important est à faire concernant la significativité des données temporelles :

Concernant les pauses, dès lors que deux touches sont frappées, l'intervalle temporel entre les deux frappes peut être décrit comme une pause. Bien évidemment, cet intervalle n'est significatif qu'au-delà d'un certain seuil, qui lui-même varie selon les préoccupations du chercheur. Les psychologues cognitivistes soucieux de décrire la charge cognitive de la frappe, par exemple (les «pauses motrices », cf. Alves, Castro \& Olive,

- les traces sont hétérogènes : parfois, elles sont très précises, parfois elles sont inexistantes. De ce fait, il n'est pas toujours possible d'éliminer toutes les solutions sauf une. » (Lebrave, 1987, p.121). 
2007) fixeront un seuil très bas (unité : milli-seconde) tandis que pour des généticiens voulant isoler de larges empans d'écriture le seuil sera évidemment beaucoup plus important (unité : seconde et 1/10ème de seconde). Accéder d'emblée à la description de toutes les pauses, c'est bien souvent se trouver confronté à des informations précises certes, mais dont on ne sait que faire parce qu'elles ne répondent pas à une interrogation de recherche.

Concernant la chronologie, se pose le même problème de significativité des données. Un exemple typique est la correction des erreurs de frappe au moment de la scription d'un mot : un scripteur écrit licvr, il revient en arrière, supprime la lettre c, puis inscrit e à la fin du mot (livre). Une des questions qui se pose est de savoir en quoi cette correction est significative, et de quoi. Dans le cas présent, elle peut servir à valider l'hypothèse d'une vigilance orthographique toujours à l'œuvre, mais encore une fois il faut savoir de quelle informations on a besoin dans tel cadre d'investigation : en quoi cette vigilance orthographique est-elle utile, par exemple, du point de vue de la création ?

Bien d'autres exemples pourraient être cités de l'écart entre l'extrême précision dont est aujourd'hui capable la technologie dans la restitution des données et la diversité des besoins des chercheurs. Cet écart implique une reconfiguration du traitement du matériau, avec dans l'idéal - c'est l'un des objets du travail de l'équipe "Manuscrits, linguistique, cognition " de l'Item - une automatisation partielle du processus. Les problèmes liés aux marques temporelles n'ont pas encore reçu de réponse satisfaisante mais ils méritaient d'être posés ici car ils sont emblématiques de ces données qui, par leur nouveauté, posent des questions encore non résolues. Ce sont maintenant certaines catégories construites par la génétique que nous allons examiner, en nous demandant dans quelle mesure elles sont opératoires pour traiter des corpus en temps réel.

\section{Les catégories de la génétique textuelle à l'épreuve des corpus en temps réel.}

Au moment de son développement dans les années 1970, la génétique textuelle s'est employée, en particulier au sein de l'équipe "Manuscrits et linguistique " de $\mathrm{l}^{\prime}$ Item $^{7}$, à forger des outils d'investigation applicables à toute écriture :

- tout manuscrit est analysable comme le résultat d'une suite de

7. L'équipe «Manuscrits et linguistique » a rassemblé les linguistes membre de l'Item jusqu'en 2005, où elle s'est scindée en trois équipes : «Linguistique du manuscrit» (responsable: Jean-Louis Lebrave), «Processus d'écriture et cognition" (resp: Jean-Louis Lebrave) et "Génétique et théories linguistiques » (resp : Irène Fenoglio). A la suite de la fusion de deux équipes, le pôle " linguistique » de l'Item comporte aujourd'hui l'équipe " Génétique et théories linguistiques" (resp: Irène Fenoglio) et l'équipe "Manuscrits, linguistique, cognition » (resp. Claire Doquet-Lacoste). 
substitutions qui sont : des ajouts, des suppressions, des remplacements et des déplacements ;

- la notion de substitution n'est pas symétrique mais nécessairement orientée par la chronologie de l'écriture, déterminante pour l'explication du processus ;

- les catégories de substitutions ${ }^{8}$, que les manuscrits ne permettent d'envisager que comme des modifications apportées à une version initiale de l'écrit, sont déterminées à l'aide de leurs traces graphiques : ratures proprement dites et ajouts en marge, en interligne, etc. Ces traces permettent d'inférer qu'une substitution a été effectuée immédiatement après l'inscription du segment sur lequel elle porte, ou bien non immédiatement.

Ceci conduit à synthétiser de la sorte les opérations d'écriture (Grésillon, 1989) :

$$
\begin{aligned}
& \text { - le remplacement: A } \square \mathrm{B} \\
& \text { - l'ajout: } \square \square \mathrm{A} \\
& \text { - la suppression: } \mathrm{A} \square \square \\
& \text { - } \quad \text { le déplacement: AX } \square \mathrm{XA} .
\end{aligned}
$$

Dans cette synthèse, le fléchage entre les éléments est signifiant : il représente le mouvement par lequel l'énoncé est transformé. Même si les éléments comparés sont des énoncés (énoncé d'origine / énoncé modifié), c'est en termes d'opérations que les choses sont décrites et ce choix correspond à une position épistémologique originale : envisager l'écriture comme un processus qui ne s'arrête pas aux états d'un écrit mais où est centrale l'activité du sujet énonciateur ; s'intéresser à ce que Louis Hay appelle « la troisième dimension du texte », «l'épaisseur de son devenir » :

[...] le sort de l'œuvre se joue avec des élans et des épuisements, des bégaiements et des vides, des ruptures et des inachèvements qui nous déroutent. Le texte ne s'abolit pas dans cette profondeur de champ, il apparaît seulement comme un objet bien plus complexe que les modèles anciens d'une œuvre, bien plus aléatoire que ses modèles actuels. (Hay, 2002, p. 58)

C'est bien là-dessus que convergent les généticiens, affirmant la relativité de l'idéal du texte ${ }^{9}$ comme celle de sa clôture ${ }^{10}$, forts du principe

8. Le terme de substitution, employé par les généticiens au moins au début de leurs travaux, pose un problème de confusion avec l'une des substitutions, le remplacement (d'ailleurs lui-même nommé, parfois, «substitution »). C'est la raison pour laquelle nous lui préférerons dorénavant le terme d'opération, qui a en outre l'avantage de mettre l'accent sur le processus plutôt que sur son résultat.

9. "Les diverses étapes de retours sur du déjà écrit ne s'inscrivent pas sur une ligne droite qui conduirait sans faille vers l'idéal du texte définitif. La vision téléologique d'une avancée de l'écriture vers l'achèvement, c'est-à-dire vers la perfection, est contredite par les manuscrits eux-mêmes." (Grésillon, 1990, p. 32)

10. "On reconnaît pour clos un texte où convergent la finitude, la finition et la finalité. La finitude est aléatoire, elle peut relever d'un arbitraire motivé après coup. La finition est le sentiment d'achèvement. Rien ne vous dit si ce 
que «ce qui a été écrit n'est pas plus prestigieux ni révélateur ni moins significatif d'avoir été barré ou biffé » (Bellemin-Noël, 1982, p. 163). Dès lors, c'est l'écriture en acte ${ }^{11}$ qui intéresse, avec son corrélat, le sujet ${ }^{12}$. Cette écriture s'affirme comme le centre de l'intérêt des chercheurs, c'est pour la décrire qu'ils mettent au point le paradigme de la génétique textuelle. Et tout de suite, un paradoxe : sur les manuscrits, ce n'est pas le mouvement de l'écriture mais son résultat, même provisoire, qui est rendu visible. L'écriture est reconstituée à partir des instantanés que constituent les différents feuillets témoignant des états intermédiaires de l'écrit. Bien évidemment l'appréhension même de l'acte d'écrire est informée du matériau et des informations que les chercheurs sont capables d'y discerner : tout en affirmant son intérêt pour l'écriture en acte, la génétique travaillant sur des manuscrits s'appuie sur des objets figés. Voici quelques exemples des limites que posent les manuscrits à l'analyse scripturale.

\subsection{L'ajout. Ajout à quoi ? Ajout de quoi ?}

Décrit par Grésillon (1989, p.178) comme la substitution qui transforme rien en quelque chose $(\square \square \mathrm{A})$, l'ajout pose des problèmes de frontières : quel est ce rien auquel on ajoute ? Le terme est réservé, dans l'étude des manuscrits, à l'inscription d'éléments graphiques à l'entour du texte central, entre les lignes, voire entre les mots, dans les espaces laissés blancs par le texte préalablement. Si les manuscrits en portent les traces, ce sont, malgré le premier terme de la formule harissienne $\square \square \mathrm{A}$, toujours des ajouts à quelque chose. L'étude génétique des manuscrits part d'un premier état de l'écrit et s'appuie sur les traces des opérations effectuées sur cet existant.

Cette catégorisation est liée au matériau étudié, qui pose comme un être-là le texte central et offre entre autres accroches pour reconstituer la chronologie de l'écriture les interventions dans les à-côtés. Grâce au positionnement des énoncés, on détermine en premier lieu deux entités : le texte central, dont l'ébauche a vraisemblablement constitué le début de l'écriture, et les entours, les marges, qui viennent moduler ce texte.

Le traitement de texte offre un espace tout différent, sans rature, malléable à l'infini, mais contraignant aussi par la linéarité qu'il impose. Qui a observé, dans les manuscrits de Valéry, l'immixtion du dessin et du

sentiment vient de celui de perfection ou de celui de lassitude. La finition est souvent l'effet d'une saturation structurale qui bloque en cours de route tout ou partie de l'œuvre. Quant à la finalité, elle est certes importante, mais les écrivains ont cent fois avoué que leur œuvre avait trahi leur projet. De sorte que la clôture d'un texte est plutôt une hypothèse d'école qu'une évidence scientifique." (Debray-Genette, 1982, p. 167)

11. Cf. le titre d'un numéro 103 de la revue Langage et Société : Ecriture en acte et genèse du texte (Fenoglio 2003).

12. «En abordant l'écriture, la critique rencontre inéluctablement une instance écrivante, tendue entre le vécu et la forme et qui parcourt cet espace de tensions par le sillage de la plume. » (Hay, 2002, p. 55) 
texte, a du mal à imaginer cet écrivain devant un ordinateur. Sur traitement de texte, sauf utilisations marginales, pas de dessin, pas de sortie de la ligne prétracée, pas de marge. Tout élément inscrit est fondu illico dans l'écrit existant, à moins de jeux typographiques bien plus difficilement réalisables au clavier qu'au stylo. Ceci signifie, du point de vue du scripteur, que toute modification s'intègre à l'énoncé comme si elle en avait toujours fait partie, étouffant ainsi le caractère historique de la constitution du texte qui fait place à l'impression d'un objet toujours propre, toujours neuf, non altéré par les aléas de son écriture. ${ }^{13} C^{\prime}$ est l'antiajout, parce que l'anti-rature, si l'on ne considère comme des ajouts que ce qui est hors linéarité. Mais dans les reconstitutions en temps réel, le lecteur / spectateur des films de l'écriture peut observer tous les mouvements du curseur, toutes les modifications apportées. La notion d'objet existant à travailler n'émerge pas ici, puisque les modifications se fondent instantanément dans le déjà écrit: il n'y a pas un texte central et des entours, pas plus qu'il n'y a un brouillon et une mise au net: on peut isoler autant d'états intermédiaires qu'on le souhaite, on peut observer toutes les opérations de constitution de ces états. Alors qu'un manuscrit donne accès au résultat d'opérations sur la mise en œuvre desquelles les informations sont extrêmement ténues, à partir d'une reconstitution en temps réel, on voit ces opérations se dérouler. Dès lors, la frontière entre ce qui est écrit et ce qui va s'écrire est mouvante, et c'est ce qui induit le caractère problématique de l'ajout: la reconstitution de l'écriture par le logiciel rend visible le fait qu'à tous les moments de la constitution du texte, des mots viennent s'ajouter à d'autres mots déjà écrits.

\section{2. Remplacement vs suppression + ajout}

C'est également à un problème de frontière que nous confronte la notion de remplacement : dans le cas d'une suppression suivie d'un ajout, quand doit-on considérer qu'il s'agit de deux opérations (une suppression simple et un ajout simple), quand faut-il identifier une seule opération (un remplacement) ? Le problème se pose déjà à partir des manuscrits : ReyDebove conclut simplement que c'est » la communauté de projet ou de contenu " qui décide de l'existence ou non d'un remplacement (ReyDebove 1982, p.114). Ce critère assez flou laisse une grande part à l'initiative personnelle du généticien et il est peu satisfaisant dès lors que l'on n'est pas dans le cas d'un remplacement quasi-synonymique. Le passage par une analyse en constituants syntaxiques paraît plus approprié puisqu'il permet de s'abstraire de considérations sur le projet du lecteur pour s'intéresser aux contraintes de fonctionnement textuel. L'équivalence syntagmatique a en outre l'avantage de se conformer aux critères de l'analyse distributionnelle, d'où sont issues les catégories d'ajout, suppression, remplacement et déplacement.

13. Il arrive que les écrivains qui utilisent le traitement de texte dessinent, mais cela se fait alors sur une sortie papier, comme l'a montré I. Fenoglio sur des manuscrits de Pascal Quignard: "Pascal Quignard. Dessins, images comme lecture "for-intérieure" de l'oeuvre à venir" colloque IMEC, Les dessin d'écrivains, 18-19 fevrier 2008 (cf. Fenoglio 2007 et Fenoglio 2009). 
Les reconstitutions en temps réel posent un problème supplémentaire : celui du remplacement en deux temps. Par exemple, dans la phrase suivante (copie d'un étudiant) :

Le cahier bleu est une œuvre méconnue mais extrêmement intéressante de L. Wittgenstein.

Sont effectuées les modifications suivantes :

- la suppression de l'expansion nominale « de L. Wittgenstein »

- puis, une heure après, le retour à cet endroit et l'ajout, exactement à la même place, de « de ce philosophe si apprécié aujourd'hui ».

Dans un manuscrit, l'énoncé se présenterait ainsi :

Le cahier bleu est une œuvre méconnue mais extrêmement de ce philosophe si apprécié aujourd'hui intéressante de L. Wittgenstein.

La modification serait classée, sans le moindre doute, comme un remplacement. Avec les données temporelles que livrent les reconstitutions en temps réel, il me semble difficile de tenir cette position : il paraît clair en effet que la suppression de A et l'inscription de B ne s'inscrivent pas dans une opération commune. A était syntaxiquement suppressible, il a été supprimé, et par la suite (même si, au niveau du sens et de la synonymie référentielle, $\mathrm{A}$ et $\mathrm{B}$ sont en relation symétrique et interchangeables), B a été ajouté. Faut-il pour autant ignorer l'équivalence référentielle et traiter les deux opérations indépendamment l'une de l'autre? Cette question renvoie au fait que fondamentalement, les opérations d'écriture consistent en des ajouts et des suppressions, et leurs combinaisons éventuelles : remplacer, tout comme déplacer d'ailleurs, $c^{\prime}$ est supprimer puis ajouter. Il convient donc peut-être de poser l'ajout et la suppression comme les deux opérations fondamentales et de considérer le remplacement (et le déplacement, puisque le même genre de cas peut échoir ${ }^{14}$ ) comme des combinaisons dont la réalisation s'effectue selon des modalités diverses : remplacement enchaîné ou remplacement en deux temps par exemple.

\subsection{Variantes textuelles et variations scripturales.}

Les premiers ouvrages de génétique textuelle travaillent la notion de variante, elle-même héritée de la tradition philologique ${ }^{15}$ : «la notion de variante suppose en principe une version considérée comme référence ;

14. Soit par exemple l'énoncé suivant : « Nous partîmes à l'aube » qui devient «A l'aube nous partîmes ». Si l'on compare les deux énoncés, on observe qu'il y a eu déplacement $\mathrm{du}$ GP, mais les choses peuvent très bien s'être déroulées ainsi : (1) inscription de "Nous partîmes à l'aube », (2) suppression de "à l'aube », (3) une heure plus tard, ajout de " A l'aube » avant « nous partîmes ». Sur le plan strictement procédural, il est alors difficile de parler de déplacement, alors que sur le plan des équivalences syntagmatiques le déplacement (GNS + GV + GP $\square$ GP + GNS + GV) est effectif.

15. Cette parenté vaut d'ailleurs à la notion même de variantes des critiques sévères : 
c'est par rapport à elle que dans une édition critique on peut établir un appareil de variantes. » (Grésillon 1994, p. 246). Si l'on ne s'intéresse qu'à l'avant-texte, on peut considérer chaque opération d'écriture comme une production de variantes, c'est-à-dire d'états différents. L'intérêt de la notion est d'englober les traces de toutes les opérations d'écriture. Sa limite principale, si l'on s'attache à l'originalité de l'approche génétique qui réside dans la tentative de comprendre l'ensemble des événements d'écriture, est son ancrage dans une problématique textuelle plus que scripturale. Pour pallier ce problème, la génétique textuelle a différencié, dans les manuscrits, la variante d'écriture de la variante de lecture. Cette différenciation est liée à l'activité supposée du scripteur au moment de la variation : la variante de lecture se produit pendant que le scripteur se relit, tandis que la variante d'écriture se produit au sein même de la scription.

Dans un manuscrit d'écrivain, la variante de lecture est repérable au fait qu'elle n'est pas effectuée sur la ligne d'écriture mais au-dessus, audessous, en marge, sur une feuille annexe, etc. ; bref, qu'elle témoigne d'un retour dans ce qui a déjà été écrit en vue de le modifier ${ }^{16}$. Ce repérage est lié au matériau dont l'analyse - qui vise la reconstitution temporelle de l'écriture - se base sur des indices essentiellement spatiaux. C'est leur répartition dans l'espace de la page qui définit les variantes (lecture ou écriture) mais elles ne peuvent pas toujours être immédiatement classées chronologiquement. L'adaptation de cette dichotomie aux reconstitutions en temps réel pose plusieurs problèmes.

Tout d'abord, l'écran ne permettant pas l'écriture en marge ou hors ligne, ce critère ne peut plus être utilisé comme distinctif des variantes de lecture. En revanche, la chronologie des opérations est, comme leur

Il est important de délimiter avec précision le domaine d'application de la variante philologique : elle concerne tous les cas - et seulement les cas - dans lesquels on a de bonnes raisons de considérer qu'il existe un texte de base unique dont on peut reconstituer la lettre à travers une série d'incarnations présentant entre elles des variations qui, correctement analysées, permettront de remonter vers le texte source. Ceci vaut par exemple pour une œuvre dont on possède une copie autorisée, mais dont l'édition a subi des transformations non attribuables à l'auteur. [...] On a dit depuis longtemps qu'il était incorrect de reprendre le modèle philologique pour décrire des documents génétiques en mettant le stemma "la tête en bas" : il n'existe aucune symétrie entre les variantes que subit un texte après sa publication et la variation parfois intense que connaît le projet textuel avant d'être livré au public. Dès lors, le domaine d'application de la notion de variante dans la critique génétique devrait être extrêmement restreint. Peut-être même faudrait-il renoncer totalement au terme (Ferrer \& Lebrave, 1991, p. 15).

16. La variante de lecture n'est pas limitée à un ajout effectué à proximité de l'unité biffée qu'elle remplace: elle peut se produire lors d'une réecriture globale du passage. Elle ne laisse pas alors de trace locale de biffure : comme pour ce qui a été dit plus haut au sujet de l'ajout, c'est la comparaison des différents états du texte qui permet d'isoler la modification, donc de parler de variante. 
ampleur, exactement enregistrée par le logiciel utilisé. Soit le début d'écriture d'un conte par une élève de Cours Moyen 2 :

- ajout de Un jour blanquette

- suppression de Un jour blanquette

- $\quad$ ajout de Un jour blanquette voulait acheter un

- suppression de un

- remplacement de voulait par alla

- ajout de un homme du nom de segin

- ajout de Elle l'emmena chez elle

Le remplacement de voulait par alla est visiblement effectué dans l'élan de la scription de la phrase. A ce titre, il me semble qu'il doit être classé comme variante d'écriture : la scriptrice n'a vraisemblablement pas eu besoin de relire sa phrase pour effectuer le remplacement, elle avait en tête le verbe qu'elle a remplacé dans un mouvement de rectification immédiate de ce qu'elle venait d'écrire.

Si l'on avait affaire au tapuscrit, il se présenterait ainsi :

alla

Un jour blanquette homme du nom de segin. Elle l'emmena chez elle.

Le remplacement de voulait par alla serait donc considéré comme une variante de lecture, ce classement étant déterminé par l'impossibilité de savoir quant exactement a eu lieu la substitution (pendant l'écriture de la phrase elle-même / après écriture du paragraphe ou du texte $)^{17}$. Avec la reconstitution en temps réel, le doute n'est pas permis quant à l'activité de la scriptrice : c'est bien en période d'écriture qu'elle effectue cette

17. Cette affirmation ne doit pas induire une vision caricaturale des résultats - très précis - obtenus lors de l'étude des manuscrits : la génétique textuelle explore toutes les voies pouvant indiquer la chronologie des opérations, et particulier elle est souvent capable de différencier les substitutions ayant eu lieu au moment de la production du texte de celles qui ont lieu une fois le texte bien avancé, ou terminé. L'examen attentif de corrélations sémantiques, entre un remplacement effectué p.1 et un élément se trouvant p.25, par exemple, aide à dater la substitution (il fallait que la p.25 soit écrite pour qu'elle puisse avoir lieu). On se fie aussi à la graphie : examinant un manuscrit de Heine, J.L. Lebrave note à propos d'un remplacement en lecture que les mots remplaçants "sont d'une écriture différente de celle de l'ensemble de la page, au trait plus fin, plus rapide»; il précise que "les données relatives aux caractéristiques de l'écriture permettent d'enrichir l'information en précisant que cette intervention est décalée dans le temps par rapport à l'écriture de l'ensemble de la page, et qu'il s'agit très probablement de corrections effectuées au cours d'une relecture d'un passage ou de l'ensemble du texte. » (1983, p.15). Ces précautions ne permettent pourtant pas de différencier à coup sûr, parmi les variantes de lecture, celles qui ont été effectuées immédiatement, au cours de l'écriture de la phrase, de celles effectuées une fois écrite la phrase (ou le paragraphe) qui les contient. 
correction ; il est intéressant de différencier ce comportement de celui qui consisterait à écrire la phrase entière puis à y revenir pour la corriger ${ }^{18}$, ce qui témoignerait vraisemblablement d'une activité de relecture concertée. Par conséquent, sans remettre en cause la validité de l'opposition variante de lecture / variante d'écriture pour les corpus manuscrits, on ne peut la transposer telle quelle aux reconstitutions de l'écriture analysées $\mathrm{ici}^{19}$.

D'autre part, nombreux sont les exemples de ce type : le scripteur écrit en fin de texte, il remonte largement dans le texte pour effectuer une correction (i.e. variante de lecture), puis revient en fin de texte pour poursuivre l'écriture. Que dire de cette continuation du texte ? Elle a de toute évidence été initiée après relecture de tout ou partie du texte, et en cela elle paraît correspondre à une variante de lecture. Toutefois, il ne s'agit pas de l'inscription de texte nouveau dans du texte déjà écrit mais de la continuation de ce texte ${ }^{20}$. Empreinte de la lecture qui l'a précédée, cette reprise de l'écriture est donc un hybride entre variante de lecture et variante d'écriture.

Dans le même ordre d'idée, que dire des ajouts dans le déjà écrit qui n'en finissent plus, les paperolles de Proust par exemple ? Ce sont des énoncés qui viennent modifier un énoncé existant, et à ce titre ils sont liés à la lecture de cet énoncé ; mais au moment de la production de ces "variantes de lecture ", la posture est-elle constante ou doit-on penser qu'à un certain moment, le scripteur est pris dans l'écriture et perd de vue qu'il est en train de modifier son écrit? Autrement dit, ces opérations constituent-elles des hybrides entre variantes de lecture et variantes d'écriture ?

De cette série de problèmes, il semble impossible de déterminer arbitrairement une règle, tant le basculement lecture / écriture dépend du

18. Néanmoins, le fait d'avoir écrit un mot et de le remplacer tout de suite par un autre n'a rien à voir avec l'écriture directe du mot définitif. J. Rey-Debove (1982) considère que toute rature implique une activité métalinguistique, opposée à la production spontanée. Cette activité métalinguistique est présente dans l'exemple donné ici : si le remplacement de voulait par alla n'est pas une variante de lecture, au sens où il n'a pas forcément nécessité de relecture pour être effectué, il reste un processus métalinguistique (une variante, au sens de l'ITEM) qui ne doit pas être traité comme un ajout spontané.

19. L'opposition entre variante de lecture et variante d'écriture est d'ailleurs questionnée, y compris à partir de corpus manuscrits, en particulier par I. Fenoglio (2003b) qui, à partir de manuscrits d'Andrée Chédid, a explicité la relation entre lecture et écriture dans la création littéraire.

20. La distinction entre ajout au sein du texte et ajout en continuation du texte, saillante sur les manuscrits, paraît devoir être conservée, avec l'intuition que ce n'est pas la même chose d'écrire dans et d'écrire après, en prolongement. Cette intuition demande évidemment à être théorisée à partir d'études empiriques. 
contexte des opérations. Mieux vaut sans doute, dans un premier temps, considérer l'opposition entre lecture et écriture comme celle de deux pôles entre lesquels les opérations viennent se positionner, étant entendu qu'il n'est jamais possible d'affirmer que cette opération-ci a été effectuée sans relecture préalable, ni que celle-là a nécessité sur le moment la relecture intégrale. Lecture et écriture se trouvent donc en relation dialectique plutôt qu'exclusive ${ }^{21}$.

\section{Implications sur le plan des modèles linguistiques à employer}

Les reconstitutions de l'écriture en temps réel permettent à l'observateur de partager une partie de l'activité du scripteur au moment de la frappe. Elle peuvent aussi lui donner l'illusion - c'est un de leurs écueils - de partager les préoccupations du scripteur. Avec ce matériau, de manière sans doute plus évidente encore qu'à partir de manuscrits, l'activité cognitive du sujet scripteur apparaît clairement comme une dimension centrale de l'écriture.

Les propositions faites plus haut sont en cohérence avec ce constat : en préconisant d'user avec prudence des termes de remplacement et de déplacement, comme de ceux de variantes de lecture et d'écriture, on met l'accent sur la dimension cognitive de l'écriture, souhaitant inclure cette dimension dans le traitement des données langagières. Dès lors, les analyses transformationnelles utilisées comme base de délimitation des opérations d'écriture (cf. 2.1), doivent s'adjoindre, comme c'est déjà le cas dans les manuscrits, le recours aux théories du sujet linguistiques portées par le courant énonciatif.

\subsection{L'écriture comme succession d'ajustements.}

Les fondateurs de l'Item et de la génétique textuelle affirmaient déjà, voici plus de trente ans, la nécessité de revenir à la prise en compte du sujet scripteur :

La conception génétique donne au texte une cause : celle du sujet écrivant. Sa présence manifeste dans le manuscrit permet de penser à nouveau le rapport du sujet à l'œuvre qui était devenu comme une aporie de la réflexion critique. (Hay, 1989, p. 19).

Le «sujet écrivant » ne se confond pas avec la personne réelle, ni avec

21. Ceci me semble en adéquation avec la conception des relations entre lecture et écriture qui prévaut à l'ITEM, dont les travaux ne peuvent laisser penser que cette distinction de mots reflète une distinction de faits : il est bien évident que la lecture fait partie intégrante de l'écriture et que la relecture de son propre texte n'est pas non plus séparable de l'écriture qui va la suivre. Almuth Grésillon cite à ce propos Martin Walser, rappelant que "lire et écrire sont une même expérience ». (1993, p.139) 
le sujet psychologique ${ }^{22}$. Ce sujet dont les manuscrits tracent la présence est l'énonciateur tel que Benveniste en a esquissé les contours et donné les modes d'accès à travers «l'appareil formel de l'énonciation »: «Le langage n'est possible que parce que chaque locuteur se pose comme sujet, en renvoyant à lui-même comme je dans son discours. " (Benveniste, 1966, p. 260). La notion d'énonciateur permet de rendre compte du fait que toute activité de langage est liée à un JE. Ce qui se joue dans l'écriture et ce dont les manuscrits comportent la trace, c'est le rapport de l'énonciateur au matériau auquel il se confronte. De ce fait, chaque variante va être envisagée, non isolément, mais en relation avec son contexte : état du texte au moment où elle intervient, activité de l'auteur, effet produit par la variante. La théorie énonciative apparaît comme le cadre adéquat de cette analyse : en posant les différentes opérations d'écriture et les niveaux sur lesquels elles portent (énonciatif, prédicatif, lexical, etc.) comme en interaction, elle permet de considérer l'écriture comme un tout et en fournit le principe unificateur ${ }^{23}$. La notion d'ajustement développée par Culioli permet de fédérer les analyses et a l'avantage de mettre l'accent sur l'articulation entre un texte et un sujet énonciateur.

Décrivant les manuscrits comme lieu d'observation de l'écriture in statu nascendi, Louis Hay met à l'épreuve les outils linguistiques pour montrer que l'opposition entre lecture et écriture est inadéquate :

[...] la genèse fait surgir des interactions qui rendent cette opposition ambiguë. Nous disons d'un terme assez singulier que l'écrivain se relit - comme s'il avait déjà eu à se lire - et nous savons bien que ces relectures déclenchent d'ordinaire autant de réécritures. (Hay, 2002, p. 83)

L'écriture est donc située comme un processus d'échange entre deux entités dont la même personne, l'auteur, assume tour à tour les fonctions de producteur et de récepteur d'un énoncé. Il n'y a évidemment pas étanchéité entre les instances lectorale et scripturale à l'œuvre dans l'écriture, puisque toutes deux sont assumées par l'auteur ${ }^{24}$ : la souplesse

22. «Le sujet de l'écriture n'a plus guère de statut dans la critique contemporaine : discrédité, dans un premier temps, par la banalité des explications biographiques, il s'est trouvé par suite refoulé hors du texte par la rigueur théorique des analyses formelles. Et cependant, il resurgit aujourd'hui au centre d'interrogations nouvelles. En abordant l'écriture, la critique rencontre inéluctablement une instance écrivante, tendue entre le vécu et la forme et qui parcourt cet espace de tensions par le sillage de la plume. » (Hay, 2002, p. 55)

23. "L'assimilation de syntaxique à formel, d'origine mathématique et licite en soi, est seulement permise en linguistique à titre de première approximation. Sinon, on en revient à l'automate, instrument aux mains d'un sujet libre qui crée et interprète, à la forme et au fond, au signifiant et au signifié, et la suite." (Culioli, 1968, p.110)

24. Les écrivains évoquent ce dédoublement, tel Louis-René Des Forêts disant : « Je suis mon propre lecteur par lequel l'auteur en moi est sans cesse tenu en 
du cadre énonciatif a permis de théoriser ces positions tout en prenant en compte la sinuosité de leur frontière. A partir de la notion linguistique de double locution, Lebrave propose celle de «double locution génétique » pour nommer l'interaction entre l'auteur-scripteur et l'auteur-lecteur, dans un cadre proche de la co-énonciation culiolienne :

[...] un même auteur se trouve démultiplié en plusieurs instances locutives : il est alternativement scripteur et lecteur, et à ces deux rôles fondamentaux, il faut sans doute en ajouter un troisième, celui de la réflexion. (Lebrave, 1987, p.770).

Dans les reconstitutions en temps réel, c'est sans doute parfois ce troisième rôle, la réflexion, que viennent matérialiser les pauses. A moins de considérer qu'une pause correspond à une activité complètement extérieure à l'écriture - ce qui est sans doute partiellement vrai mais ne peut être systématique - on peut envisager la pause comme le lieu de la réflexion, de la relecture, de la projection du texte à venir. La précision chronologique permet également d'appréhender plus finement le rapport du scripteur à son écrit, en fonction du moment de l'écriture. Prenons le cas d'un scripteur ayant commencé une production et se remettant à l'ouvrage quelques jours après. Il se trouve tout d'abord en position d'auteur-lecteur (relecteur du texte écrit), et comme l'a montré LamotheBoré (1998) la distance temporelle entre l'écriture première et la relecture crée une sorte d'extériorité : auteur-lecteur certes, le sujet énonciateur est d'abord davantage lecteur d'un texte qu'il reconnaît au cours de sa lecture tout en en étant un peu étranger. Dès lors, les modifications qu'il va effectuer sont celles d'un auteur qui, étant le même individu que celui qui a produit le premier jet, est en même temps différent, tout à la fois en soi une semaine s'est écoulée - et dans son rapport au texte. Bien mieux que les manuscrits, qui disent si une modification s'est faite au fil de la plume ou a posteriori mais ne donnent que très peu d'indications sur la distance temporelle entre une inscription et sa modification, les reconstitutions de l'écriture en temps réel mettent en évidence le caractère dilaté du temps de l'écriture. Elles invitent par conséquent à affiner la notion de double locution génétique, en $\mathrm{y}$ introduisant la dilatation temporelle de l'énonciation écrite.

\section{2. Les non-coïncidences langagières comme cadre d'analyse des modifications.}

Culioli affecte au langage un caractère de non-extériorité au sujet, en parlant de "relation complexe d'extériorité-intériorité » (1968, p.108). Ce faisant, il pose l'hétérogène comme constitutif de l'énonciation. Le caractère foncièrement hétérogène $\mathrm{du}$ langage, travaillé de manière extrêmement précise et complète par Jacqueline Authier-Revuz (1995),

bride » ou Aragon lançant : "Je n'ai jamais écrit mes romans, je les ai lus. » (cités par Grésillon, 1993, p. 139). 
apparaît à travers les ratures des manuscrits comme dans les modifications textuelles que montrent les reconstitutions en temps réel : on peut analyser les modifications comme des traces des non-coïncidences langagières (non-coïncidence interlocutive, interdiscursive, des mots aux choses, des mots à eux-mêmes) même si, en l'absence d'énoncé commentatif, il serait abusif de parler de modalisation autonymique ${ }^{25}$. Il est extrêmement difficile d'interpréter, dans un manuscrit, la raison d'être des modifications, puisque pour chacune les possibilités sont multiples. Avec la précision chronologique, les reconstitutions en temps réel rendent possible le recensement des enchaînements d'opérations qui permettent parfois de préciser l'enjeu des modifications.

Les données temporelles permettent également $\mathrm{d}$ 'affiner la caractérisation des modifications de l'énoncé du point de vue de la distance entre inscription première et rectification. Les ajustements effectués au cours de l'écriture entretiennent un rapport temporel variable avec l'énoncé qu'ils modifient. Dans le cas des boucles méta-énonciatives étudiées par Authier-Revuz, la rectification - ou le commentaire sur l'énoncé premier - sont immédiats. Cette caractéristique est liée au fait qu'Authier-Revuz a étudié des énoncés à partir de leur réception : énoncés oraux où émission et réception sont simultanés (et où donc, nécessairement, les commentaires méta-énonciatifs se font sur ce qui vient juste d'être dit), énoncés écrits dont elle n'a pas cherché à explorer la genèse (où énoncé premier et commentaire sont contigus dans le texte, sans que l'on sache rien de leur contiguïté ou non contiguilté dans l'énonciation). Il est évident que l'accès aux opérations en temps réel permet de cerner le rapport entre modalités énonciatives et énoncé final, en particulier à travers l'examen des modifications selon qu'elles sont plus ou moins différées, temporellement et spatialement, de l'inscription initiale de l'énoncé modifié. Ce n'est pas la même chose de modifier un terme qui a été inscrit 30 secondes plus tôt et de modifier le même terme plusieurs heures, voire plusieurs jours après son inscription première, après la relecture complète, etc. Les reconstitutions en temps réel questionnent la notion même de variante de lecture / variante d'écriture

25. Authier-Revuz a travaillé sur des énoncés de structure $X$, je dis $X^{\prime}$ où $X^{\prime}$ est l'autonyme de $X$, caractérisés comme des «boucles méta-énonciatives de modalisation autonymique. Ces énoncés s'accompagnent souvent de gloses commentatives que l'auteur a classées et dont elle a fait émerger les 4 noncoïncidences : interlocutive, interdiscursive, des mots aux choses, des mots à eux-mêmes. Ces énoncés commentatifs, qui relèvent du secteur du métalinguistique (épilinguistique chez Culioli), révèlent les représentations spontanées du langage mais aussi les modalités de l'énonciation et attestent qu'elle ne va pas de soi : «ainsi le secteur spécifique de méta(épi)-linguistique inscrit dans une structure méta-énonciative doit-il être saisi à la fois en tant que discours spontané de représentation du langage, et en tant que rouage de l'énonciation, et, plus précisément, en tant que discours spontané comme rouage de l'énonciation. » (Authier-Revuz, 1995, p.21). 
en montrant que lecture et écriture sont véritablement inséparables, comme sont inséparables l'énonciation et la modalisation de l'énoncé ${ }^{26}$. Elle mettent en évidence le dédoublement de l'énonciateur, à la fois et indissociablement scripteur et lecteur, souvent dans une posture de réticence par rapport à son énoncé, comme le montrent par exemple des faits, non marginaux, d'énonciation où l'on a l'impression qu'au moment de l'inscription première le scripteur sait déjà que l'énoncé ne conviendra pas mais l'inscrit tout de même - souvent à un rythme très lent, avec des pauses longues entre les mots -- pour le supprimer à peine terminé (Doquet-Lacoste, 2004 : 364).

La précision des données temporelles permet également de caractériser l'écriture comme une activité inscrite dans une dilatation du temps de l'énonciation. Les différents retours sur le dit se concrétisent par des mouvements du curseur et/ou des rectifications et modifications textuelles plus ou moins différées dans le temps et éloignées du lieu de travail initial. Cette énonciation dilatée fait apparaître des phénomènes d'attraction dont l'étude permet de cerner un peu mieux la part de la langue, matérialisée par le discours déjà déjà écrit, dans la création de texte. Deux types d'attraction ont été empiriquement constatés jusqu'ici, dans des corpus d'écriture en temps réel :

Attraction sémique : ce type d'attraction s'observe dans des périodes de relecture, à la faveur de remplacements lexicaux mettant en jeu une isotopie. C'est le cas par exemple dans une narration de souvenir par une élève de $6^{\text {ème }}$, avec une série de remplacements mettant en place une isotopie qui fait passer le texte d'une narration réaliste, se donnant comme fidèle au vécu, à une narration nettement subjectivée (Doquet-Lacoste, 2002).

Attraction morpho-syntaxique: dans le cadre de l'écriture d'une phrase complexe, la structure «comment + verbe pronominal » apparaît comme un moule syntaxico-lexical mis en place sans doute par hasard mais dont la récurrence va constituer un guidage de l'écriture (DoquetLacoste, accepté).

La diversité des éléments observés, si elle n'a pas encore permis d'élaborer une représentation unifiée, permet au moins d'affirmer la nécessité de n'écarter, pour l'analyse, aucun niveau linguistique et par conséquent aucun modèle. L'étude de l'écriture met en évidence la nécessité d'observer conjointement au détail d'une opération la globalité $\mathrm{du}$ texte telle qu'elle se présente au scripteur au moment de ladite opération, la logique du texte, mais également le contexte temporel et

26. La modalisation d'un énoncé se fait parfois avant même son énonciation, comme à l'oral : « je viens de vivre une - je dirai - une belle journée ». Dans le cadre de la lecture, l'usage des guillemets a également une fonction de modalisation à l'avance, par exemple dans l'incipit de Un Amour de Swann: «pour entrer dans le «petit noyau», le «petit groupe», le «petit clan» des Verdurin, une condition était suffisante mais elle était nécessaire $[\ldots]$ » 
chronologique, les opérations qui précèdent, ce que l'on pourrait appeler la logique de l'action. Sans cette double prise en compte, il est extrêmement difficile de comprendre la raison d'être d'une opération scripturale. Cette articulation entre vue globale et examen détaillé des faits d'écriture abonde encore la nécessité de ne rejeter aucun paradigme linguistique mais de les penser en complémentarité.

\section{Pour conclure, pour commencer...}

Les recherches prenant en compte les données temporelles de l'écriture débutent. Elles s'appuient sur des outils, principalement des logiciels de traitement de texte, qui sont encore largement perfectibles et dont le rendu des données demande à être travaillé pour permettre des analyses linguistiques. Ces recherches constituent un enjeu important pour les sciences du langage, confrontées à la nécessité d'articuler les paradigmes en multipliant les regards - morphologique, syntaxique, sémantique, énonciatif - sur des faits de détail tout en ancrant l'analyse dans la double logique de l'action et du texte. Ce à quoi devrait aboutir toute analyse de reconstitution scripturale, c'est la mise au jour de la logique de l'écriture, décrite linguistiquement. Cette logique réside dans la compréhension de la structuration du texte et de l'enchaînement des opérations scripturales, qui tout en s'appuyant sur des données langagières et processuelles livrées à l'observation ne peut faire abstraction du sujet qui les produit. En affirmant la nécessité de s'en tenir aux traces et d'exploiter les données au plus près de leur matérialité, le point de vue exposé ici est celui de l'énonciation «au sens restreint ${ }^{27}$, une approche énonciative qui se garde de la tentation sur-interprétative tout en explorant au plus loin les indices de la subjectivité et de l'appropriation par le sujet énonciateur de la langue et de son fonctionnement. Puisque c'est un sujet qui utilise la langue, les données ne sont interprétables hors cette situation. Dans le cadre des modèles exposés plus haut, en particulier les ajustements énonciatifs de Culioli et les non-coïncidences langagières d'Authier-Revuz, il s'agit d'observer l'écriture et d'en déterminer les unités descriptives pour construire, à partir de la mise en évidence des logiques d'écritures singulières, les traits distinctifs de l'énonciation scripturale.

27. « Par énonciation au sens restreint, j'entends, à la suite de Culioli, l'ensemble des opérations régulières de constitution-interprétation des énoncés appréhendables par référence aux paramètres linguistiques - c'est-à-dire nécessairement idéalisés - constitutifs de la situation d'énonciation (énonciateur, co-énonciateur, moment de l'énonciation); j'oppose une telle approche à celle de l'énonciation au sens large, qui tend à être co-extensive au champ entier de la pragmatique, et à inclure la variabilité des facteurs individuels et des circonstances particulières de la situation de communication effective entre un émetteur et un récepteur, les déterminations extralinguistiques de la signification (connaissance d'univers, représentations, etc.) » Fuchs, 1994, p.85. 


\section{Eléments de bibliographie}

Alamargot, D., Lambert, E. \& Chanquoy, L. (2005), La production écrite et ses relations avec la mémoire. Approche Neuropsychologique des Acquisitions de l'Enfant, 17, 4146.

Alves, R.-A., Castro, S.-L., Olive, T. (2007), “Execution and pauses in writing narratives : processing time, cognitive effort ain typing skill", International Journal of Psychology.

Authier-Revuz, J. (1995), Ces Mots qui ne vont pas de soi. Boucles réflexives et noncoïncidences $d u$ dire, Paris, Larousse, 1995.

Cohen, M., Crasson, A. \& Fenoglio, I. (2005), Récit d'Edmond Jabès. Les cinq états du manuscrit. Paris, Textuel.

Chesnet, D., Alamargot, D. (2005), « Analyse en temps réel des activités oculaires et grapho-motrices du scripteur. Intérêt du dispositif Eye and Pen », L'Année psychologique, $\mathrm{n}^{\circ} 105$ (3).

Culioli, A. (1990) Pour une linguistique de l'énonciation : opérations et représentation, tome 1, Paris, Ophrys.

(2002), Variations sur la linguistique, Paris, Klincksieck.

Dédéyan, A., Largy, P., \& Negro, I. (2006), " Mémoire de travail et détection d'erreurs d'accord verbal : étude chez le novice et l'expert », Langages, 164, 57 70 .

Doquet-Lacoste, C. (2002), «Et pourtant, ils écrivent! Etude génétique de productions scripturales d'enfants de CM2 », Modernités, n¹8, L'auteur entre biographie et mythographie, Bordeaux, Centre de Recherche sur les Modernités Littéraires, Presses Universitaire de Bordeaux, 173-186.

(2004) Etude génétique de l'écriture d'élèves de Cours Moyen 2, année 1995-96, Thèse de Doctorat de Sciences du Langage soutenue à l'Université de Paris 3 le 17 juin 2003, Lille : Atelier National de Reproduction des Thèses.

(2006) « L'objet insaisissable : quelques considérations sur l'analyse de l'écriture sur traitement de texte », Genesis, n²7. 35-44.

(accepté) «Entre énonciation «spontanée » et effets stylistiques, place de la parataxe dans la production de textes à l'école », in Béguelin, M.-J., Avanzi, M. \& Corminboeuf, G. (éds) Actes du colloque La parataxe, Neuchâtel, 12-15 février 2007. À paraître.

Favart, M. \& Olive, T. (2005), «La production écrite: Apprentissage et fonctionnement expert », Psychologie Française, 50-3.

(ed.) (2003a), Ecriture en acte et genèse du texte. Langage et société, 103.

(2003b), «La fin de Lucy d'Andrée Chedid: un explicit de la verticalité humaine », Genesis 21, 2003, 141-159.

(2007), »Fête des Chants du Marais, un conte inédit de Pascal Quignard. Genèse in vivo et traitement de texte », in Genesis 27, éd. Jean-Michel Place, p. 73-95.

(2009), "Textual Genetics and Manuscript in the text processing. A new definition of a text? Essay on the avant-texte of Pascal Quignard's novel" Belgian Journal of Linguistics, 23 : New approaches in text linguistics (S. Mellet et D.Longeet eds), p. 45-61.

Ferrer, D. , Lebrave, J.-L. (1991), « De la variante textuelle au geste d'écriture variant », L'Ecriture et ses doubles, Paris, CNRS, p.9-25.

Fuchs C. (1994), Paraphrase et énonciation, Paris, Ophrys. 
Grésillon, A. (1989), « Fonctions du langage et genèse du texte », in Hay, L. (éd.) La Naissance du texte, Paris, José Corti.177-192.

Grésillon, A. (1993), "Méthodes de lecture», in Hay, L. (ed) Les Manuscrits des Ecrivains, p.138-161.

Grésillon, A., Lebrave, J.-L. (1982b), «Les Manuscrits comme lieu de conflits discursifs », in Fuchs, C., Grésillon, A., Lebrave, J.-L. et al., La Genèse du texte : les modèles linguistiques, Paris, CNRS, coll. Textes et manuscrits.129-175.

Hay, L. (2002), La Littérature des écrivains. Questions de critique génétique, Paris, José Corti.

Lamothe-Boré, C. (1998), Choix énonciatifs dans la mise en mots de la fiction : le cas des brouillons scolaires, Thèse de doctorat de Sciences du Langage, Université Stendhal-Grenoble 3.

Leblay, C. (2007), «L'avant-texte comme texte sur le vif. Analyse génétique d'opérations d'écriture en temps réel ", Langue française $n^{\circ} 155$, Paris, larousse, p. 101-113

Lebrave, J.-L. (1987), Le Jeu de l'énonciation en allemand d'après les variantes manuscrites des brouillons de H. Heine. Thèse de Doctorat d'Etat, Université de ParisSorbonne.

(2001), « Comment écriront-ils ? », Diogène, n¹96, oct-déc 2001, p.163-171.

Rey-Debove, J. (1982), « Pour une lecture de la rature », in Fuchs, C., Grésillon, A., Lebrave, J.L. et al., La Genèse du texte : les modèles linguistiques. Paris : CNRS. 2172. 